\title{
Physicochemical Characterization of Alginate Beads Containing Sugars and Biopolymers
}

\author{
Tatiana Aguirre Calvo ${ }^{1}$ and Patricio Santagapita ${ }^{1,2}$ \\ ${ }^{1}$ Industry Department and Organic Chemistry Department, Faculty of Exact and Natural Sciences, \\ University of Buenos Aires (FCEN-UBA), Buenos Aires, Argentina \\ ${ }^{2}$ National Council of Scientific and Technical Research (CONICET), Buenos Aires, Argentina \\ Correspondence should be addressed to Patricio Santagapita; prs@di.fcen.uba.ar
}

Received 29 June 2016; Accepted 14 August 2016

Academic Editor: Yi-Hung Chen

Copyright (C) 2016 T. Aguirre Calvo and P. Santagapita. This is an open access article distributed under the Creative Commons Attribution License, which permits unrestricted use, distribution, and reproduction in any medium, provided the original work is properly cited.

\begin{abstract}
Alginate hydrogels are suitable for the encapsulation of a great variety of biomolecules. Several alternatives to the conventional alginate formulation are being studied for a broad range of biotechnological applications; among them the addition of sugars and biopolymers arises as a good and economic strategy. Sugars (trehalose and $\beta$-cyclodextrin), a cationic biopolymer (chitosan), an anionic biopolymer (pectin), and neutral gums (Arabic, guar, espina corona, and vinal gums) provided different characteristics to the beads. Here we discuss the influence of beads composition on several physicochemical properties, such as size and shape, analyzed through digital image analysis besides both water content and activity. The results showed that the addition of a second biopolymer, $\beta-\mathrm{CD}$, or trehalose provoked more compact beads, but the fact that they were compact not necessarily implies a concomitant increase in their circularity. Espina corona beads showed the highest circularity value, being useful for applications which require a controlled and high circularity, assuring quality control. Beads with trehalose showed lower water content than the rest of the system, followed by those containing galactomannans (espina corona, vinal, and guar gums), revealing polymer structure effects. A complete characterization of the beads was performed by FT-IR, assigning the characteristics bands to each individual component.
\end{abstract}

\section{Introduction}

Considerable research has been conducted in the field of encapsulation leading to studies on the protection and controlled release of bioactive compounds used in pharmaceutical, cosmetic, and food industry $[1,2]$. There has been great interest in the use of hydrogel beads that contained encapsulated agents, being the search of adequate excipients that assure stability and controlled release a great challenge. Several hydrocolloids and biopolymers are used for this purpose due to their properties related to wall protection and delivery as well as their functional and sensory attributes. Among them, sodium alginate (isolated from brown algae) is one of the most used excipients for formulating hydrogel beads due to its encapsulating/gelling properties when cross-linked with divalent ions like calcium [3], leading to a huge range of possibilities, since it possesses several advantages compared with other commonly used biopolymers listed below: (a) easy and low-cost preparation method, which consists of dropping an aqueous solution (or emulsion) of the biopolymer and bioactive compound into $\mathrm{CaCl}_{2}[4,5]$; (b) compatibility with other excipients, increasing its wall and release properties; (c) versatility, by allowing encapsulation of a wide range of bioactive compound, which are highly sensitive to environmental conditions, since the interior is known to be chemically inert [6]; (d) nontoxicity and biodegradability, since results of in vivo studies demonstrated the development of a biodegradable alginate carrier system for antibiotics and bone cells, providing a potential treatment procedure for infected bone defects [7]. However, some disadvantages are often associated with this carrier, including high biomolecule leakage, low mechanical strength, and large pore size [8].

In order to optimize the encapsulation efficiency and control release of biocompounds, the addition of sugars and biopolymers arises as a good and economic strategy. Among sugars, trehalose, a disaccharide used as cryo- and 
dehydroprotective agent, has been proven to improve the formulation and protect enzymes during freezing and thermal treatment on alginate beads $[5,9]$, and $\beta$-cyclodextrin, cyclic oligosaccharides composed of seven glucopyranose units, possesses the ability to encapsulate hydrophobic molecules with suitable size inside their cavity to form inclusion complexes [10]. Among the biopolymers, chitosan is one of the most used, since a cationic biopolymer provides thicker membranes for the direct interaction with alginate, modifying also some thermal [5] and functional properties [11]. An anionic biopolymer such as pectin, similarly to alginate, forms hydrogel following the egg-box model [12], competing for $\mathrm{Ca}$ (II) with alginate to form the gelation network, affecting functional properties [5]. It is known that the inclusion of food gums on mixtures of emulsions to form beads has advantages showing a more rigid structure and at the same time protects the compound from diffusion [13]; then, the inclusion of four neutral gums was assessed: guar and Arabic gums, which are two of the most used gums, and two novel gums, vinal and espina corona gums. The latter gums are novel nongelling biopolymers that provide high viscosity to aqueous solutions and are relatively stable against variations in $\mathrm{pH}$, ionic strength, and temperature [14-16].

The purpose of the present work was to assess the influence of beads composition on several physicochemical properties, such as size and shape, analyzed through digital image analysis, as well as both water content and activity. A complete characterization of the beads was performed by FT-IR, assigning the characteristics bands to each one of the individual components.

\section{Materials and Methods}

2.1. Materials. The employed materials are listed below: sodium alginate (Algogel 5540) Cargill SA (San Isidro, Buenos Aires, Argentina), MW $=1,97.10^{5} \mathrm{~g} / \mathrm{mol}$ and mannuronate/ guluronate ratio $=0.6 ;$ D-trehalose dihydrate (Hayashibara Co., Ltd., Shimoishii, Okayama, Japan/Cargill Inc., Minneapolis, Minnesota, USA), MW $=378 \mathrm{~g} / \mathrm{mol} ; \beta$-cyclodextrin $(\beta-C D)$ (Amaizo American Maize-Products Co., Hammond, IN, USA), $\mathrm{MW}=1185 \mathrm{~g} / \mathrm{mol}$; low methoxyl pectin $(L M)$, Meath \& Nutrition Degussa Argentina Inc. (San Isidro, Buenos Aires, Argentina) with esterification degree between 26 and 31\% and amidation degree between 16 and 19\%; guar gum (Cordis SA, Villa Luzuriaga, Buenos Aires, Argentina), $\mathrm{MW} \sim 220,000 \mathrm{~g} / \mathrm{mol}$ and $\mathrm{M} / \mathrm{G}$ (mannose/galactose $)=1.8$; Arabic gum (Biopack, Zárate, Buenos Aires, Argentina). $\mathrm{MW} \sim 250,000 \mathrm{~g} / \mathrm{mol}$ and purity of $99 \%$; espina corona gum was provided from Supply Idea Argentina S.A. (Chaco, Argentina), with the following percentage composition: moisture, $0.04 \mathrm{~g} / 100 \mathrm{~g}$; ashes, $1.44 \mathrm{~g} / 100 \mathrm{~g}$; fat, $0.27 \mathrm{~g} / 100 \mathrm{~g}$; protein, $2.17 \mathrm{~g} / 100 \mathrm{~g}$; crude fiber, $0.70 \mathrm{~g} / 100 \mathrm{~g}$; polysaccharides, $85.38 \mathrm{~g} / 100 \mathrm{~g}$; and $\mathrm{M} / \mathrm{G}=2.5$ [16]; chitosan, medium molecular weight viscosity of $200-800 \mathrm{cP}(1 \% \mathrm{w} / \mathrm{v}$ in $1 \%$ acetic acid at $25^{\circ} \mathrm{C}$, Brookfield viscometer), and degree of deacetylation of $75-85 \%$ (Sigma-Aldrich Chemie $\mathrm{GmbH}$, Steinheim, Germany). Vinal gum is a gum extracted from seeds of Prosopis ruscifolia as reported by Busch et al. [14], with $\mathrm{M} / \mathrm{G}=1.6$, protein content of $1.9 \pm 0.2 \%(\mathrm{w} / \mathrm{w})$, and $\mathrm{MW}$ of $1.43 \cdot 10^{6} \mathrm{Da}$.

Extra virgin olive oil (Molino Cañuelas SACIFIA, Mendoza, Argentina) was purchased in the local market to formulate emulsions. The emulsions were prepared in an UltraTurrax T18B (IKA-Werke GMBH \& CO.KG, Staufen, Germany) at 15,500 rpm (speed 4) for a period of $10 \mathrm{~min}$. The oil was emulsified with the corresponding biopolymer solution in a mass ratio of $1: 2$.

2.2. Preparation of Alginate Beads. The formulations of initial emulsions were $1 \%(\mathrm{w} / \mathrm{v})$ alginate with $20 \%(\mathrm{w} / \mathrm{v})$ trehalose mixed with $0.25 \%(\mathrm{w} / \mathrm{v})$ of pectin, guar, Arabic, vinal, or espina corona gums.

Alginate solutions were mixed with the biopolymers to generate initial emulsions. For all the formulation a $1 \%(\mathrm{w} / \mathrm{v})$ solution of sodium alginate was prepared with bidistilled water in a magnetic stirrer allowing full hydration of the alginate for about $12 \mathrm{~h}$; to remove any air bubbles the solution was sonicated for $3 \mathrm{~min}$. Beads were prepared by ionotropic gelation according to the drop method described previously $[5,9]$. Briefly, to ensure bead generation a $2.5 \%$ (w/v) $\mathrm{CaCl}_{2}$ solution (with trehalose $20 \%(\mathrm{w} / \mathrm{v})$, in addition to $1.5 \%(\mathrm{w} / \mathrm{v})$ $\beta$-cyclodextrin or $0.5 \%(\mathrm{w} / \mathrm{v})$ chitosan for the correspondent systems) was prepared in bidistilled water as an external medium. By dripping $10 \mathrm{~mL}$ of the solution of the initial emulsion was performed using a peristaltic pump with model BT50-1J JY10 head (Boading Longer Precision Pump Co., Ltd., China), always with constant stirring, a standard pipette tip for $0.1-10 \mu \mathrm{L}(0.35 \mathrm{~mm}$ diameter $)$ connected to a silicone tube $1 \mathrm{~mm}$ internal diameter ( $3 \mathrm{~mm}$ external) over $100 \mathrm{~mL}$ of $\mathrm{CaCl}_{2}$ solution.

The systems were generated at $\mathrm{pH}$ higher than 4 , considering the $\mathrm{pk}_{\mathrm{a}}$ values of alginate (3.38 and $\left.3.65[5,9]\right) \cdot \mathrm{pH}$ values were between 5.5 and 6 for $\mathrm{A}$ and $\mathrm{A}-\mathrm{T}$ beads and between 4 and 5 for the rest of the beads.

2.3. Size and Shape through Digital Image Analysis. The size and shape of the beads were obtained by analyzing the digital images captured by a digital camera (Canon digital camera, 3.2 Mpix PowerShotA70; Canon Inc., Malaysia; with zoom fixed at $3 \mathrm{x}$ ) coupled to a binocular microscope (Unitron MS, Unitron Inc., New York, USA; magnification at 7x). The pictures were analyzed with the free license software ImageJ. Once beads images are capture for all formulations, the size and shape were obtained with the image analysis, processing with free license software ImageJ (http://rsbweb.nih.gov/ij/). The performed analysis is shown in Figure 1.

Area, perimeter, Feret's diameter, and circularity were analyzed for at least 60 beads by applying the "analyze particle" command of the software. Feret's diameter corresponds to the longest distance between any two points along the bead boundary. Circularity is defined as a value between 0 and 1 indicating how closely the shape of the particle resembles a circle. The effect of composition on Feret's diameter and circularity were analyzed by 1-way ANOVA with Tukey post test using Prism 5 (GraphPad Software Inc.). The significant differences between values with $p<0.05$ were indicated on 


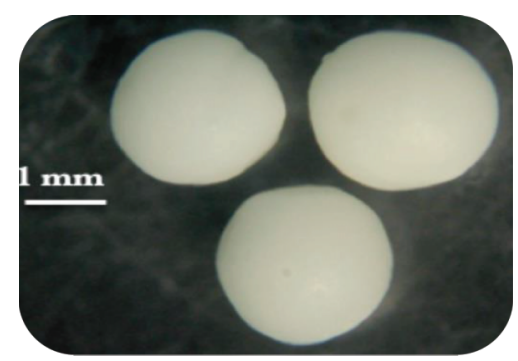

Original image (RGB)

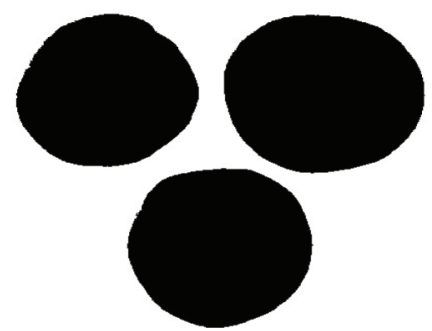

(1) Change image type to 8-bit

(2) Thresholded using

Image $>$ Adjust $>$ Threshold

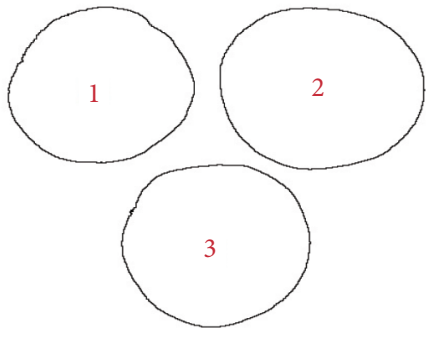

Analyze $>$ analyze particles selecting outlines: each bead is numbered and analyzed by the software

Set scale option (in analyze menu) allows the presentation of result in calibrated units (as $\mathrm{mm}$ )

FIGURE 1: Sequence of image analysis from the original image to the particle analysis. For example, beads containing only alginate (A) as biopolymer.

columns by different letters, "a" being the higher value. The statistical analysis was adapted from $[5,9,14]$. The ImageJ software was calibrated to transform the measured pixels in length units $(\mathrm{mm})$ by taking pictures of a caliper section.

2.4. Water Content and Water Activity. The total water content of the beads was determined gravimetrically by difference in weight before and after drying in vacuum oven for $48 \mathrm{~h}$ at $96 \pm 2^{\circ} \mathrm{C}$. The water content was expressed in percentage of grams of water per gram of sample (wet basis, w.b.).

Water activity $\left(a_{w}\right)$ was determined by means of an Aqualab instrument (Decagon Devices, Inc., USA).

Water content and $a_{w}$ were measured in triplicate. The effect of composition on water content and $a_{w}$ was analyzed by 1-way ANOVA with Tukey post test using Prism 5 (GraphPad Software Inc.). The significant differences between values with $p<0.05$ were indicated in columns by different letters.

\subsection{Attenuated Total Reflectance-Fourier Transform-Infrared} Spectroscopy (FT-IR). FT-IR spectra were measured on a Spectrum 400 spectrometer FT-IR/FT-NIR (Perkin Elmer, Inc., Shelton, CT, USA). Wet beads were subjected to freezedrying prior to analysis due to the high absorption of water which strongly dominates the spectrum, masking biopolymers and sugars bands. Sugars and biopolymers solutions were also freeze-dried to obtain control spectra for each excipient. All the samples were studied in a MIRacle attenuated total reflectance (ATR) accessory (PIKE Technologies, Inc., Madison, WI, USA) with a diamond/ZnSe crystal of single reflection at an incident angle of $45^{\circ}$. Two beads were placed directly thereon, without prior sample preparation, obtaining a very good signal to noise ratio. The spectra were acquired in reflection mode between 675 and $4000 \mathrm{~cm}^{-1}$, acquiring 32 scans averaged with a resolution of $4 \mathrm{~cm}^{-1}$. A strong apodization was used, with a magnitude phase correction. A flat tip was employed to obtain an intimate contact between sample and crystal, without pressure control. A background spectrum was recorded in air (without sample) prior to each spectrum measurement. Spectral analysis was performed using the Spectrum software version 6.3.5
(PerkinElmer, Inc.). The average of triplicates for each system was reported. Baseline was corrected and the spectra were normalized (between 0 and 1 ) for figure presentation.

\section{Results and Discussion}

3.1. Size and Shape through Digital Image Analysis. Alginate macrobeads of different formulations obtained by the drop method were analyzed by image analysis. The size and morphological characterization of the beads are relevant since they could have impact on different physicochemical properties such as water sorption and release of the encapsulated agent as well as consumer's acceptance (improve the aesthetic quality which could be a desirable characteristic for pharmaceutical, food, and feed products) [17]. Figure 2 shows size (determined as Feret's diameter), circularity, area, and perimeter of the beads analyzed by optical microscopy and a digital image processing technique.

The addition of any excipient to alginate resulted in significant reduction of the diameter and the area and perimeter of the beads, as shown in Figure 2. Alginate beads showed a significant deviation from sphericity (circularity values of 0.6), showing characteristics of an ellipse. The addition of any excipient to alginate caused an increase in the circularity (Figure 2(b)), except for trehalose in A-T beads, since there are no significant differences between them (A versus A-T). This increment of bead circularity due to the addition of a second biopolymer or $\beta$-CD was probably related to changes in the surface tension/viscosity of the droplets containing the initial emulsion, which was further used to form alginate beads.

It is interesting to note that the addition of a second biopolymer, $\beta-\mathrm{CD}$, or trehalose provoked more compact beads, but the fact that they were compact not necessarily implies a concomitant increase in their circularity. For example, AVG-T beads showed a smaller diameter compared to AT ones (being both diameters smaller than A beads), showing no significant differences in circularity from each other. Beads containing Arabic gum or $\beta$-CD were shown to be the smaller ones for all the analyzed parameters (area, perimeter, 


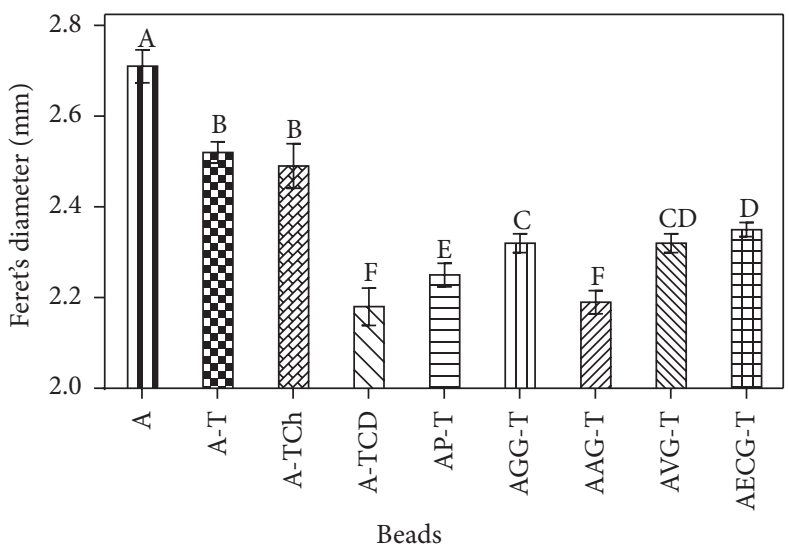

(a)

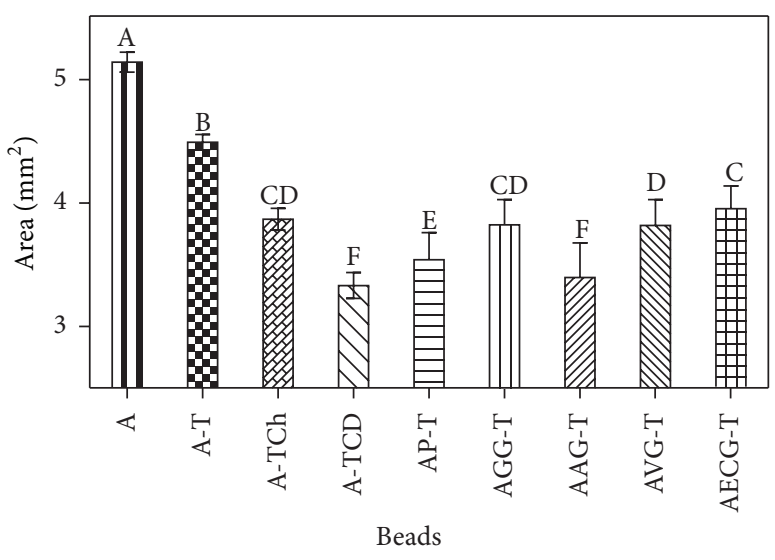

(c)

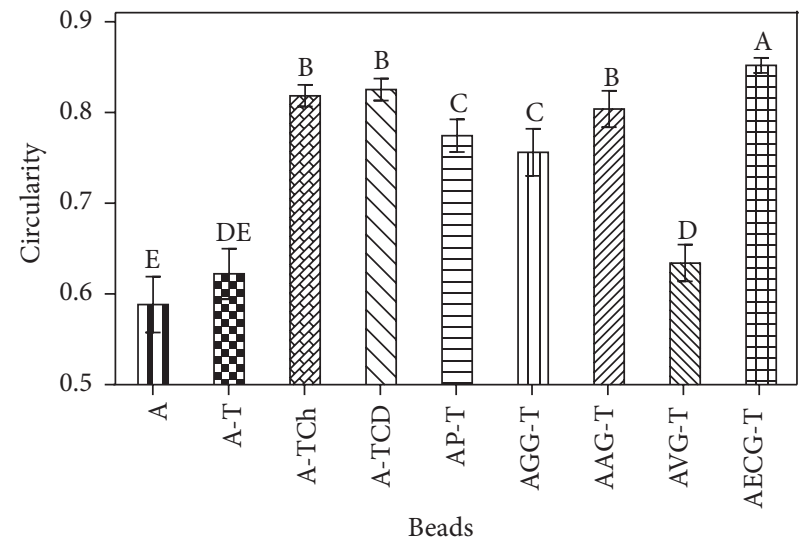

(b)

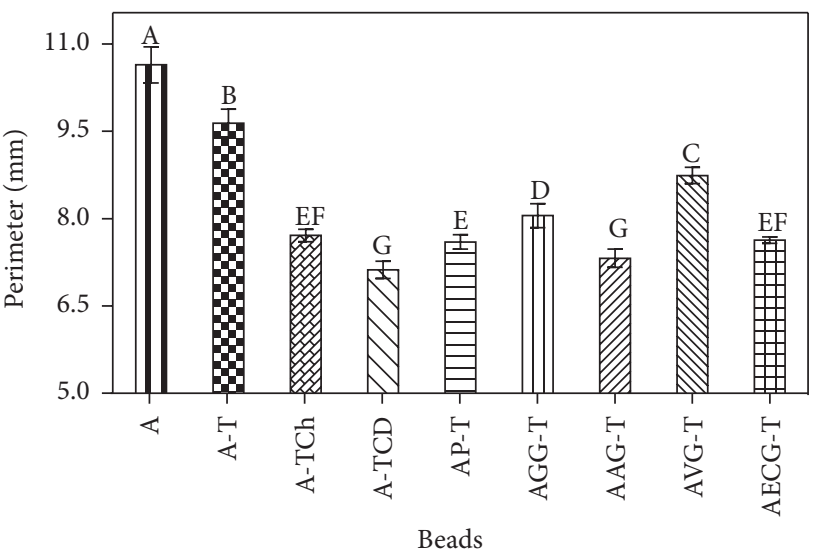

(d)

Figure 2: Feret's diameter (a), circularity (b), area (c), and perimeter (d) obtained by image analysis of the beads of different composition. Confidence intervals at $95 \%$ are indicated. At least, 60 beads of each system were analyzed. Different letters on the columns (A-G, being highest to lowest values, resp.) indicate significant differences between values with $p<0.05$.

or diameter). Beads containing espina corona gum showed the highest value of circularity (0.85).

3.2. Water Activity and Content. It is critical to know the water content and water activity $\left(a_{w}\right)$ of beads to define the appropriate processing and storage conditions, considering the high influence of water on stability when formulations containing bioactive compounds are stored [9]. $a_{w}$ and the water content were analyzed according to the composition of the beads, as shown in Table 1. As expected, the wet beads showed high $a_{w}$ values between 0.96 and 0.98 but lower than the values reported by other authors for alginate beads (around 0.99) $[9,18]$, probably due to the presence of oil in the beads. Most of the beads containing sugars and biopolymers had lower $a_{w}$ values than A beads (except for A-TCh and AP-T beads) showing that the addition of other soluble components caused a decrease in $a_{w}$. However, not all the beads had lower water content values than A beads. This difference is possible due to the fact that the beads of different systems probably had differences in their water sorption isotherms, which were not determined in this study. The beads with trehalose (A$\mathrm{T}$ ) showed lower water content than the rest of the system, followed by those containing galactomannans (espina corona, vinal, and guar gums).

3.3. Component Identification by FT-IR. The individual components of the extract and of the beads were determined by FT-IR using a micro-ATR (attenuated total reflectance) accessory. The advantage of this device is that it allows the direct measurement of the beads, without the need to use dispersants matrices (such as the commonly used $\mathrm{KBr}$ ), simplifying sample preparation and subsequent processing of data.

Figures 3 and 4 show the spectra of the freeze-dried beads and the correspondent freeze-dried controls of each employed excipient. The signals corresponding to the presence of oil are clearly seen in all beads spectra (Figures 3 and 4 ), confirming the correct incorporation of the emulsions in the beads. In these spectra, characteristic bands associated with lipid groups were observed between 1380 and 1370, 1485 and $1430 \mathrm{~cm}^{-1}$ ( $\mathrm{CH}$ bending), $1745 \mathrm{~cm}^{-1}(\mathrm{C}=\mathrm{O}$, ester) and 3010 , and 2920 and $2852 \mathrm{~cm}^{-1}$ (CH stretch) and a major band at $1160 \mathrm{~cm}^{-1}$. Besides, it is possible to observe the presence of each of the different excipients used in each bead system from the recognition of the corresponding major signals as well 
TABLE 1: Water content (as percent of wet basis, WC \% w.b.) and water activity $\left(a_{w}\right)$ obtained for different beads formulations. Standard deviation values $(n=3)$ are included.

\begin{tabular}{lcc}
\hline Beads & WC $(\%$ w.b. $)$ & $a_{w}$ \\
\hline A & $44 \pm 2^{(\mathrm{a})}$ & $0.983 \pm 0.002^{(\mathrm{a})}$ \\
A-T & $37 \pm 1^{(\mathrm{d})}$ & $0.975 \pm 0.002^{(\mathrm{b})}$ \\
A-TCh & $41 \pm 2^{(\mathrm{a}, \mathrm{b})}$ & $0.981 \pm 0.004^{(\mathrm{a}, \mathrm{b})}$ \\
A-TCD & $41 \pm 1^{(\mathrm{a}, \mathrm{b})}$ & $0.979 \pm 0.002^{(\mathrm{a}, \mathrm{b})}$ \\
AP-T & $39 \pm 3^{(\mathrm{a}, \mathrm{b}, \mathrm{c}, \mathrm{d})}$ & $0.981 \pm 0.002^{(\mathrm{a})}$ \\
AGG-T & $39 \pm 1^{(\mathrm{b}, \mathrm{c}, \mathrm{d})}$ & $0.967 \pm 0.003^{(\mathrm{c})}$ \\
AAG-T & $41.6 \pm 0.6^{(\mathrm{a})}$ & $0.958 \pm 0.006^{(\mathrm{c}, \mathrm{d})}$ \\
AVG-T & $40 \pm 2^{(\mathrm{a}, \mathrm{b}, \mathrm{c}, \mathrm{d})}$ & $0.960 \pm 0.003^{(\mathrm{d})}$ \\
AECG-T & $37.1 \pm 0.6^{(\mathrm{c}, \mathrm{d})}$ & $0.961 \pm 0.007^{(\mathrm{c}, \mathrm{d})}$ \\
\hline
\end{tabular}

Different letters (a-d) indicate significant differences between values in each column with $p<0.05$, assigning (a) to the highest and (d) to the lowest values.

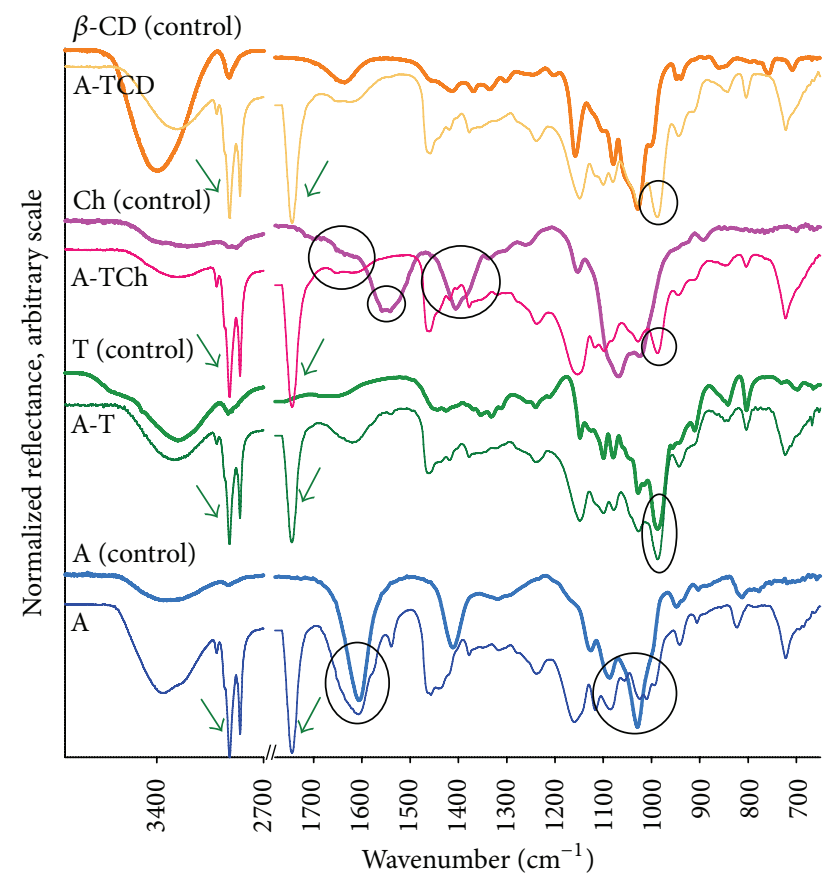

FIGURE 3: Normalized spectra obtained from freeze-dried beads and their control (freeze-dried biopolymers or sugars). Characteristics bands are highlighted by circles and arrows (lipids).

as the already reported interaction between chitosan and alginate $[5,18]$ through peak shifts in A-TCh beads (Figure 3 ).

The characteristic bands of alginate correspond to its carboxylic groups at 1595 and $1405 \mathrm{~cm}^{-1}$ (symmetric and asymmetric stretching, resp.) $[19,20]$ and have been observed also in A beads spectra. These signals are less intense in the rest of the beads spectra (Figures 3 and 4) due to the presence of trehalose, whose signals strongly dominate the spectra (bands at $983 \mathrm{~cm}^{-1}$ and $1148 \mathrm{~cm}^{-1}$, corresponding to the glycosidic bond, [21]). The band at $1033 \mathrm{~cm}^{-1}$ corresponding to $\mathrm{CO}$ stretching is characteristic of alginate as well [20,21]. The signals at $1490-1380 \mathrm{~cm}^{-1}$ correspond to both alginate and oil.

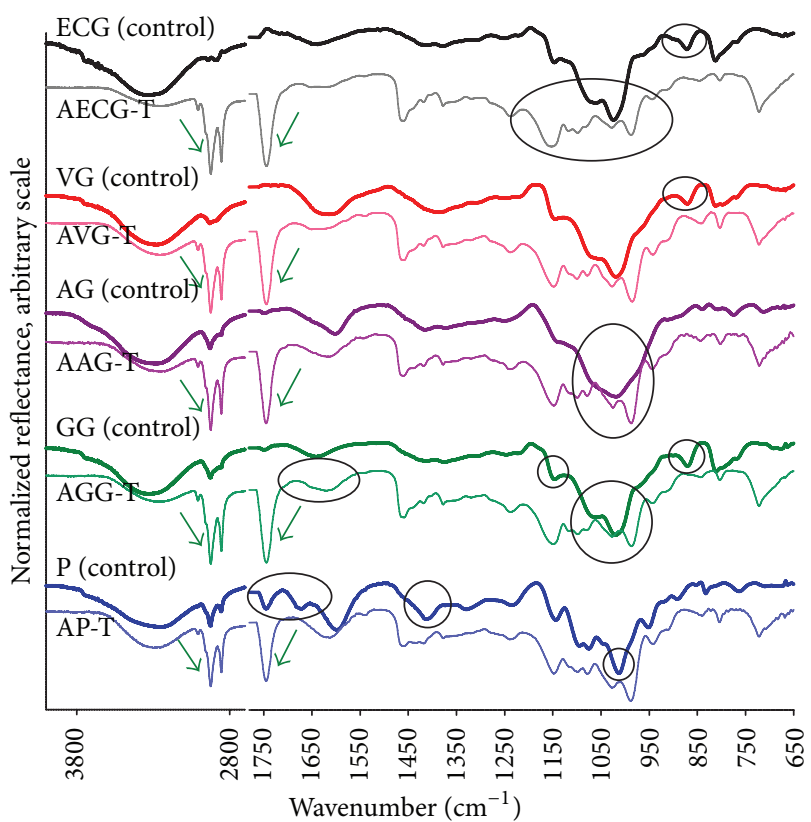

FIGURE 4: Normalized spectra obtained from freeze-dried beads and their control (freeze-dried biopolymers or sugars). Characteristics bands are highlighted by circles and arrows (lipids).

A signal shift was observed for the signal at $1033 \mathrm{~cm}^{-1}$, which generated a characteristic three peaks pattern at 1024, 1007, and $992 \mathrm{~cm}^{-1}$.

It is important to consider that the employed biopolymers present some similarities (hydrocolloid behavior, major composition of sugars, and low protein content (except for Arabic gum)). These similarities lead to similar bands in the IRspectra, but some characteristic signals (detailed below) still can be observed. Besides, the $1500-900 \mathrm{~cm}^{-1}$ region was not disturbed by the presence of water and it is very rich in terms of structural information, as many characteristic functional groups of saccharides signals appeared in this spectral region [21].

Chitosan spectrum shows an absorption peak marked at $1550 \mathrm{~cm}^{-1}$ due to the protonation of the amino group, one at $1635 \mathrm{~cm}^{-1}$ due to the amide bond, and the deformation of the $\mathrm{CH}_{2}$ groups occurring at $1405 \mathrm{~cm}^{-1}[20,22]$. The evidence of the interaction between alginate and chitosan can be seen in the A-TCh beads in the displacement and broadening of these bands $\left(1641-1610 \mathrm{~cm}^{-1}\right.$ and $\left.1405-1420 \mathrm{~cm}^{-1}\right)$, although the last one is superposed due to contribution of oil band.

Signals characteristics of pectin are $1741(\mathrm{C}=\mathrm{O}$ vibration of the $\mathrm{COOH}$ ), 1605 (symmetric vibration of COO-), 1410 (antisymmetric vibration of COO-), and $1011 \mathrm{~cm}^{-1}$ (stretching $\mathrm{C}=\mathrm{O})[23,24]$. Unlike what was observed with the chitosan, for pectin beads (AP-T), there was no displacement of bands because both were negatively charged and do not form a complex (as expected). However, the AP-T beads show more intense bands at 1415 and $1330 \mathrm{~cm}^{-1}$ (which are quite small in the scale presented) than the AT beads possibly due to the contribution of pectin. 
The spectra of gums, especially for the three galactomannans (guar, espina corona, and vinal gums), showed similarities in the carbohydrate region $\left(1200-700 \mathrm{~cm}^{-1}\right)$, especially in the signals corresponding to the anomeric carbons of the different monomers (specific signals for galactose $(\alpha$ o $\beta)$ at $871 \pm 7 \mathrm{~cm}^{-1}$, mannose $\left(876 \pm 9 \mathrm{~cm}^{-1}\right)$, and glucose $\left(915,874\right.$, and $\left.\left.767 \pm 8 \mathrm{~cm}^{-1}\right)\right)$ [25-27]. The relative intensity of bands at 1150,1070 , and $970 \mathrm{~cm}^{-1}$ with respect to the major band at $1020 \mathrm{~cm}^{-1}$ was related to the differences in the mannose/galactose ratios of the employed galactomannans. The main bands of guar gum were observed at 1017, 1082, and $1158 \mathrm{~cm}^{-1}$ (C-OH stretching), at $1648 \mathrm{~cm}^{-1}$ (observed as a shoulder in AGG-T), and at $1620 \mathrm{~cm}^{-1}$ (OH bending). Furthermore, AGG-T beads showed a broadening of the band at $1150 \mathrm{~cm}^{-1}$. Arabic gum spectrum showed characteristic bands at 3000-3600 cm $\mathrm{cm}^{-1}$ (O-H stretching), 2993 and $2918 \mathrm{~cm}^{-1}$ (C$\mathrm{H}$ stretching), and $1612 \mathrm{~cm}^{-1}(\mathrm{C}=\mathrm{O}$ stretching) with a main peak at $1017 \mathrm{~cm}^{-1}$.

To the best of our knowledge, FT-IR spectra of vinal and espina corona gums are reported for the very first time. Vinal gum spectrum showed a very broad signal between 1700 and $1500 \mathrm{~cm}^{-1}$, which includes several peaks with maxima between 1600 and $1630 \mathrm{~cm}^{-1}$. In the AVG-T beads, the shape of the broad band at $1620 \mathrm{~cm}^{-1}$ was more alike to that of vinal gum than that of alginate, showing a relative increase at $1640 \mathrm{~cm}^{-1}$ (in comparison to the band at $1620 \mathrm{~cm}^{-1}$ ). A widening of the signal at $1150 \mathrm{~cm}^{-1}$ (coincident with the presence of a band in the espina corona control spectrum) and a relative modification of the ratio of the three bands pattern (region 1060-1130 $\mathrm{cm}^{-1}$ ) were observed for the beads containing espina corona gum (Figure 4).

\section{Conclusions}

Shape and size of the beads were strongly affected by the composition. The addition of a second biopolymer, $\beta$ cyclodextrin, or trehalose causes more compact beads, but this does not necessarily imply an increase in circularity. Beads with Arabic gum or $\beta$-CD were shown to be the smaller ones. Beads containing espina corona showed the highest value of circularity, which could be useful for applications which require a controlled and high circularity, assuring quality control.

The addition of sugars and neutral biopolymers produced lower $a_{w}$ values than plain alginate beads. However, there were no concomitant lower water content values. This difference is possible due to the fact that the beads of different systems probably had differences in their water sorption isotherms. Then, it is important to keep in mind that the addition of excipients could also have impact on other physicochemical characteristics that, in turn, could affect the physicochemical properties most commonly analyzed.

The obtained results represent the starting point to overcome the disadvantages associated with alginate as a carrier, leading to a higher stability and controlled release of the alginate- $\mathrm{Ca}$ (II) beads by using a second biopolymer, $\beta$ cyclodextrin, or trehalose.
Components of the beads were successfully determined by infrared spectroscopy studies of Fourier transform (FTIR). This study allows us to observe the interaction between chitosan and alginate and the presence of each of the components used in the preparation of the beads from the identification of its main bands.

\section{Abbreviation List}

$\begin{array}{ll}\text { A: } & \text { Alginate } \\ \text { T: } & \text { Trehalose } \\ \text { Ch: } & \text { Chitosan } \\ \text { CD: } & \beta \text {-Cyclodextrin } \\ \text { P: } & \text { Pectin } \\ \text { GG: } & \text { Guar gum } \\ \text { AG: } & \text { Arabic gum } \\ \text { VG: } & \text { Vinal gum } \\ \text { ECG: } & \text { Espina corona gum. }\end{array}$

\section{Competing Interests}

The authors declare that there is no conflict of interests regarding the publication of this paper.

\section{Acknowledgments}

The authors acknowledge the financial support of ANPCYT (PICT 2013 no. 0434 and PICT 2013-1331) and CONICET and UBA (Project UBACyT 20020130100443BA). They also acknowledge Dr. Pilar Buera for the useful discussions and Dr. Verónica Busch for vinal gum donation.

\section{References}

[1] B. Zeeb, A. H. Saberi, J. Weiss, and D. J. McClements, "Retention and release of oil-in-water emulsions from filled hydrogel beads composed of calcium alginate: impact of emulsifier type and pH," Soft Matter, vol. 11, no. 11, pp. 2228-2236, 2015.

[2] B. Zeeb, A. H. Saberi, J. Weiss, and D. J. McClements, "Formation and characterization of filled hydrogel beads based on calcium alginate: factors influencing nanoemulsion retention and release," Food Hydrocolloids, vol. 50, pp. 27-36, 2015.

[3] M. H. L. Ribeiro, C. Afonso, H. J. Vila-Real, A. J. Alfaia, and L. Ferreira, "Contribution of response surface methodology to the modeling of naringin hydrolysis by naringinase Ca-alginate beads under high pressure," LWT-Food Science and Technology, vol. 43, no. 3, pp. 482-487, 2010.

[4] E.-S. Chan, B.-B. Lee, P. Ravindra, and D. Poncelet, "Prediction models for shape and size of Ca-alginate macrobeads produced through extrusion-dripping method," Journal of Colloid and Interface Science, vol. 338, no. 1, pp. 63-72, 2009.

[5] P. R. Santagapita, M. F. Mazzobre, and M. D. P. Buera, "Invertase stability in alginate beads: effect of trehalose and chitosan inclusion and of drying methods," Food Research International, vol. 47, no. 2, pp. 321-330, 2012.

[6] L. G. Griffith, "Polymeric biomaterials," Acta Materialia, vol. 48, no. 1, pp. 263-277, 2000.

[7] S. W. N. Ueng, M. S. Lee, S.-S. Lin, E.-C. Chan, and S.-J. Liu, "Development of a biodegradable alginate carrier system for antibiotics and bone cells," Journal of Orthopaedic Research, vol. 25 , no. 1, pp. 62-72, 2007. 
[8] M. M. M. Elnashar, E. N. Danial, and G. E. A. Awad, "Novel carrier of grafted alginate for covalent immobilization of inulinase," Industrial and Engineering Chemistry Research, vol. 48, no. 22, pp. 9781-9785, 2009.

[9] P. R. Santagapita, M. F. Mazzobre, and M. P. Buera, "Formulation and drying of alginate beads for controlled release and stabilization of invertase," Biomacromolecules, vol. 12, no. 9, pp. 3147-3155, 2011.

[10] P. A. Ponce Cevallos, M. P. Buera, and B. E. Elizalde, "Encapsulation of cinnamon and thyme essential oils components (cinnamaldehyde and thymol) in $\beta$-cyclodextrin: effect of interactions with water on complex stability," Journal of Food Engineering, vol. 99, no. 1, pp. 70-75, 2010.

[11] P. R. Santagapita, M. F. Mazzobre, and M. P. Buera, "Stabilization and controlled release of invertase through the addition of trehalose in wet and dried alginate-chitosan beads," in Water Stress in Biological, Chemical, Pharmaceutical and Food Systems: ISOPOW '11, G. Gutiérrez, G. Barbosa-Cánovas, L. Alamilla, E. Para-Arias, and M. P. Buera, Eds., pp. 353-360, Springer, 2015.

[12] I. Braccini, R. P. Grasso, and S. Pérez, "Conformational and configurational features of acidic polysaccharides and their interactions with calcium ions: a molecular modeling investigation," Carbohydrate Research, vol. 317, no. 1-4, pp. 119-130, 1999.

[13] I. Roy, M. Sardar, and M. N. Gupta, "Cross-linked alginate-guar gum beads as fluidized bed affinity media for purification of jacalin," Biochemical Engineering Journal, vol. 23, no. 3, pp. 193198, 2005.

[14] V. M. Busch, A. A. Kolender, P. R. Santagapita, and M. P. Buera, "Vinal gum, a galactomannan from Prosopis ruscifolia seeds: physicochemical characterization," Food Hydrocolloids, vol. 51, pp. 495-502, 2015.

[15] V. M. Busch, F. Loosli, P. R. Santagapita, M. P. Buera, and S. Stoll, "Formation of complexes between hematite nanoparticles and a non-conventional galactomannan gum. Toward a better understanding on interaction processes," Science of the Total Environment, vol. 532, pp. 556-563, 2015.

[16] M. J. Perduca, M. J. Spotti, L. G. Santiago, M. A. Judis, A. C. Rubiolo, and C. R. Carrara, "Rheological characterization of the hydrocolloid from Gleditsia amorphoides seeds," LWT-Food Science and Technology, vol. 51, no. 1, pp. 143-147, 2013.

[17] B.-B. Lee, P. Ravindra, and E.-S. Chan, "Size and shape of calcium alginate beads produced by extrusion dripping," Chemical Engineering and Technology, vol. 36, no. 10, pp. 1627-1642, 2013.

[18] L. Deladino, P. S. Anbinder, A. S. Navarro, and M. N. Martino, "Encapsulation of natural antioxidants extracted from Ilex paraguariensis," Carbohydrate Polymers, vol. 71, no. 1, pp. 126134, 2008.

[19] M. G. Sankalia, R. C. Mashru, J. M. Sankalia, and V. B. Sutariya, "Papain entrapment in alginate beads for stability improvement and site-specific delivery: physicochemical characterization and factorial optimization using neural network modeling," AAPS PharmSciTech, vol. 6, no. 2, pp. E209-E222, 2005.

[20] B. Sarmento, D. Ferreira, F. Veiga, and A. Ribeiro, "Characterization of insulin-loaded alginate nanoparticles produced by ionotropic pre-gelation through DSC and FTIR studies," Carbohydrate Polymers, vol. 66, no. 1, pp. 1-7, 2006.

[21] M. Kačuráková and M. Mathlouthi, "FTIR and laser-Raman spectra of oligosaccharides in water: characterization of the glycosidic bond," Carbohydrate Research, vol. 284, no. 2, pp. 145157, 1996.
[22] B. Smitha, S. Sridhar, and A. A. Khan, "Chitosan-sodium alginate polyion complexes as fuel cell membranes," European Polymer Journal, vol. 41, no. 8, pp. 1859-1866, 2005.

[23] A. Sinitsya, J. Čopíková, V. Prutyanov, S. Skoblya, and V. MacHovič, "Amidation of highly methoxylated citrus pectin with primary amines," Carbohydrate Polymers, vol. 42, no. 4, pp. 359-368, 2000.

[24] T. H. Kim, Y. H. Park, K. J. Kim, and C. S. Cho, "Release of albumin from chitosan-coated pectin beads in vitro," International Journal of Pharmaceutics, vol. 250, no. 2, pp. 371-383, 2003.

[25] S. Paul and G. S. Mittal, "Regulating the use of degraded oil/fat in deep-fat/oil food frying," Critical Reviews in Food Science and Nutrition, vol. 37, no. 7, pp. 635-662, 1997.

[26] H. T. Pedersen, L. Munck, and S. B. Engelsen, "Low-field ${ }^{1} \mathrm{H}$ nuclear magnetic resonance and chemometrics combined for simultaneous determination of water, oil, and protein contents in oilseeds," Journal of the American Oil Chemists' Society, vol. 77, no. 10, pp. 1069-1076, 2000.

[27] W. S. Price, "Pulsed-field gradient nuclear magnetic resonance as a tool for studying translational diffusion: part 1. Basic theory," Concepts in Magnetic Resonance, vol. 9, no. 5, pp. 299-335, 1997. 


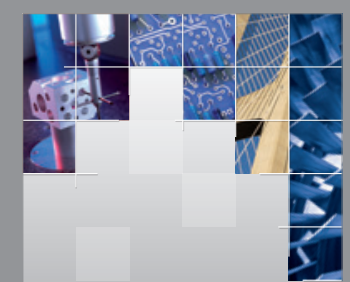

\section{Enfincering}
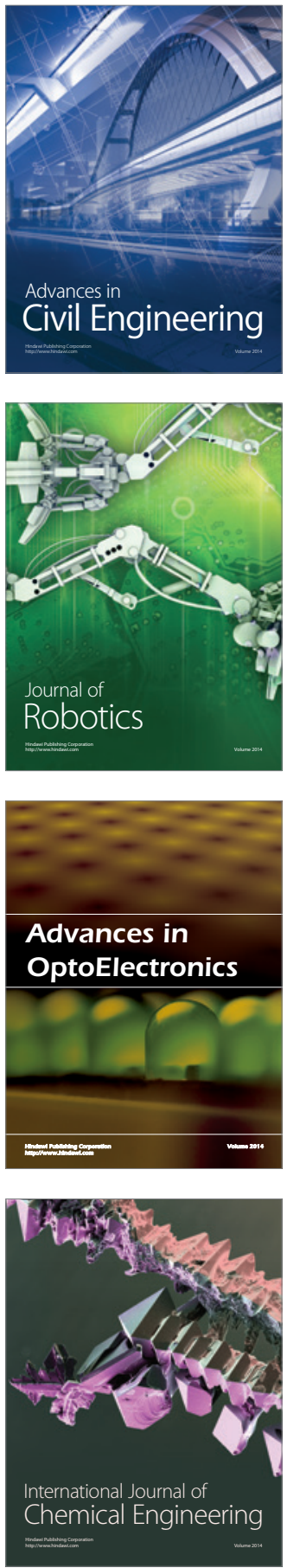

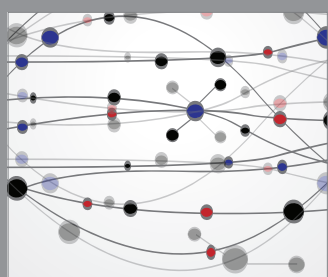

The Scientific World Journal

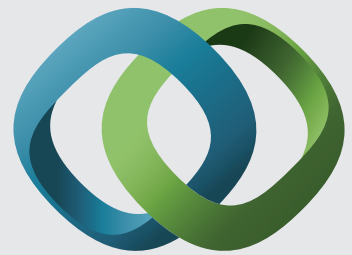

\section{Hindawi}

Submit your manuscripts at

http://www.hindawi.com
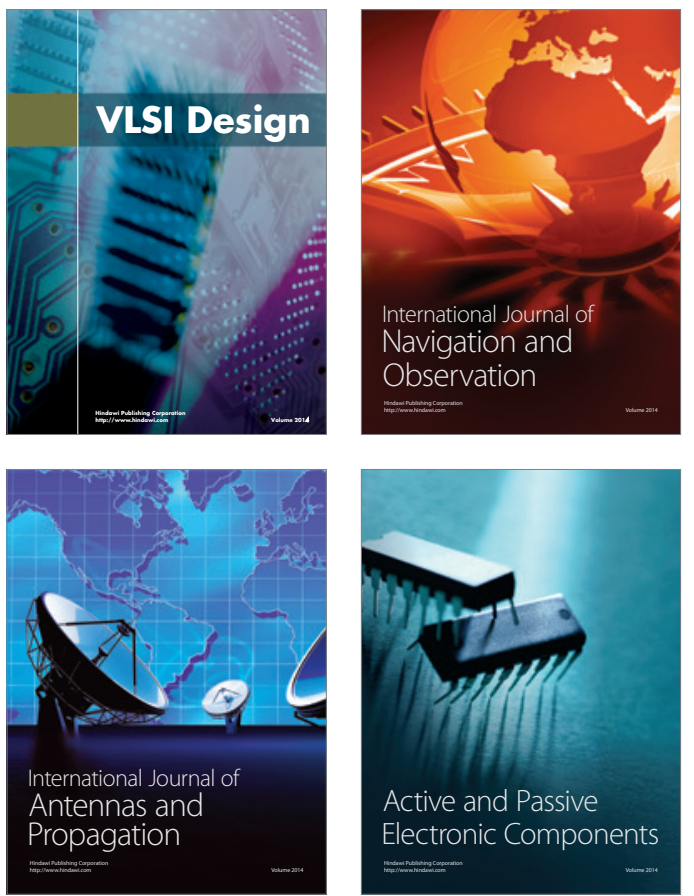
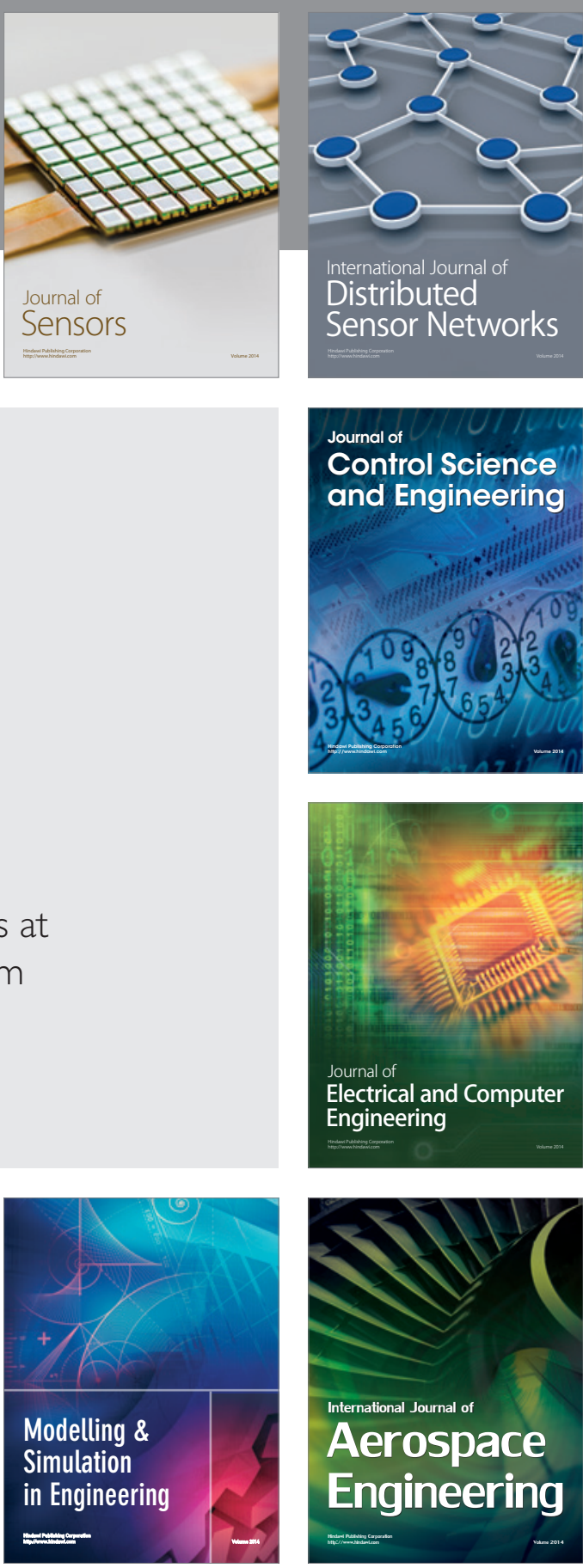

International Journal of

Distributed

Sensor Networks

Journal of

Control Science

and Engineering
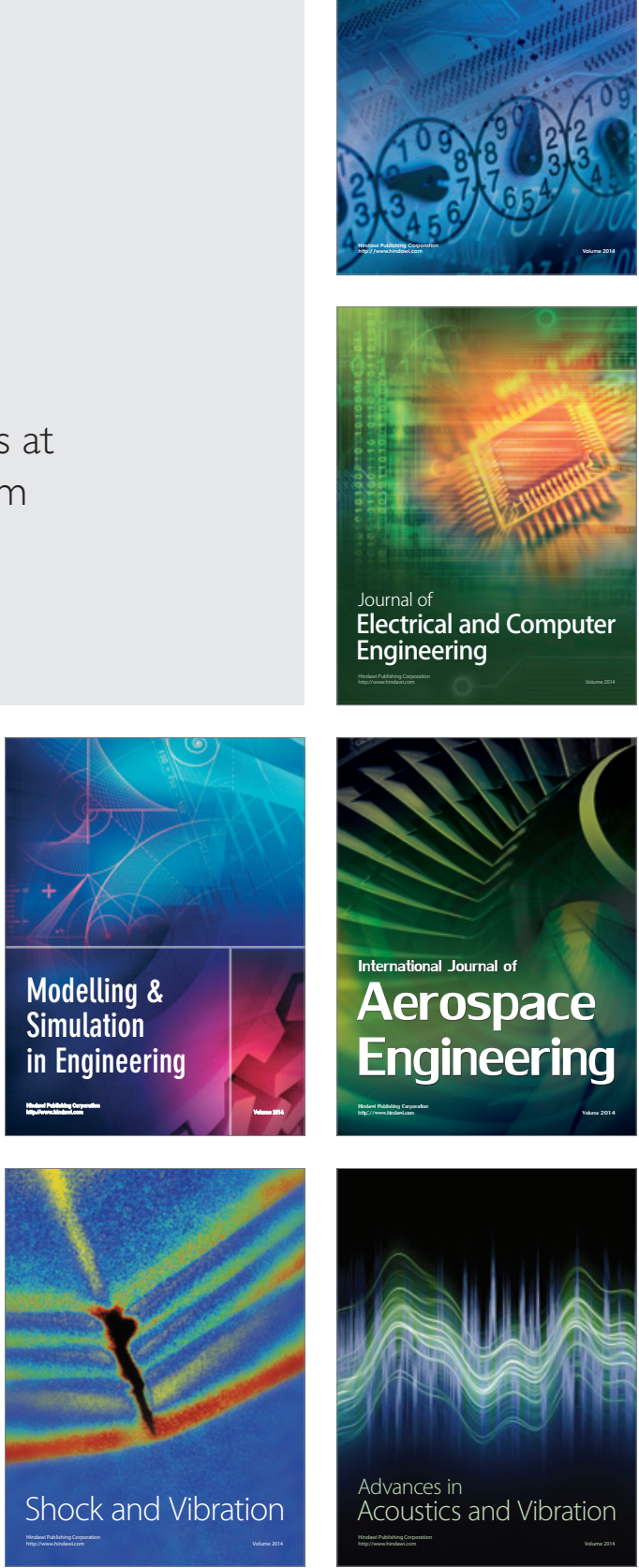